\title{
Contracts for the Management of a Non -Renewable Resource under Asymmetric Information and Structural Price Breaks*
}

\author{
David MartimorT ${ }^{\dagger}$ Jerome Pouyet ${ }^{\ddagger}$ Francesco Ricci ${ }^{\S}$ \\ This version: December 6, 2018. \\ accepted for publication on \\ Annales d'Economie et de Statistique / Annals of Economics and Statistics
}

\begin{abstract}
We characterize the optimal contract for resource extraction in a context where the concessionaire has private information on the initial stock of resource. The dynamics of extraction is characterized by a virtual Hotelling rule in which costs of extraction are replaced with virtual costs of extraction. We analyze how structural breaks in the price of resource impact the dynamics of extraction.
\end{abstract}

KEYWORDS. Non-Renewable Resource Management, Delegated Management, Optimal Contract, Asymmetric Information.

JEL CODES. Q31; D82.

\footnotetext{
${ }^{*}$ We thank Katheline Schubert for having suggested us to work on this topic and participants to the "Shale Gas and Energy Transition" Workshop held at CEPREMAP, Paris for comments related to a companion paper. This project has benefited from the financial support of CEPREMAP. All remaining errors are ours. We gratefully acknowledge the excellent research assistance of Louis Daumas.

${ }^{\dagger}$ Paris School of Economics (EHESS). E-mail: david.martimort@psemail.eu.

‡THEMA-CNRS, ESSEC Business School, Université de Cergy-Pontoise. E-mail: pouyet@essec.edu.

${ }^{\S}$ CEE-M, Univ Montpellier, CNRS, INRA, Montpellier SupAgro, Montpellier, France. E-mail: francesco.ricci@umontpellier.fr.
} 


\section{INTRODUCTION}

The SHAlE BOOM. According the U.S. Energy Information Administration (EIA (2015)), fossil fuels will still meet $87 \%$ of energy needs in 2025 and will remain the dominant source of energy supply for the 21st century. Natural gas is an alternative to other sources due to its abundance and its lower environmental damages.

In the U.S. gas trapped in shale rocks started to be exploited on an industrial scale in the 2000s. At that time, soaring oil and gas prices and increasing scarcity of conventional gas in North America made the exploitation of new plays profitable. An effort in research and development targeting access to unconventional hydrocarbon sources since the 1980s was supported by the U.S. government, including partnerships with vanguard companies such as Mitchell Energy. As breakthrough innovation in production technology emerged in the early 2000s, the exploitation of shale gas exploded. The improved extraction techniques combined hydraulic fracturing and horizontal drilling. ${ }^{1}$ Such developments lead to a massive increase in the production of, first, gas and, then, oil as from 2006 in North America. Shale gas completely flood the local market, especially after 2004, offsetting the depletion of conventional natural gas. In 2017, shale gas represented 57\% of total domestic gas production. This positive supply shock has led North America to be ranked as the second region having the lowest costs for energy and raw materials in the world after the Middle East (EIA (2015)).

Before the mid-2000s, natural gas prices were tightly linked to those of oil and their price dynamics were accordingly quite similar. However, from 2004 on, prices in North America deviated from the global trajectory of rising prices: leaving aside the 2005 and 2008 spikes due to exceptional climate, prices reached a plateau in 2004. Since 2008 U.S. natural gas prices fell, as a result of a combination of demand and supply factors. On the one hand, the abrupt and persistent negative demand shock followed from the subprime crisis. On the other hand, the tremendous expansion of the natural gas supply reflected the shale gas boom: the U.S. natural gas production in April 2016 is $40 \%$ higher than in April 2006 (EIA (2015)). This led natural gas prices in North America to a 15-years low by 2016.

Shale oil boom is also playing a role in oil price dynamics. Although the latter reflects worldwide shocks due to the global nature of the oil market, the potential supply from the exploitation of shale oil became clear in the last few years. Shale oil production underpinned growth of U.S. crude oil output by over $65 \%$ in the 7 years following April 2009. Joskow (2015) observes retrospectively that "the complementary development of

\footnotetext{
${ }^{1}$ Additional specificities of the U.S. contribute to explain the emergence of this new technology. The country hosts massive shale plays. American academic and industrial organizations host much of the expertise on geology and hydrogeology. Domestic production of conventional gas and oil attained a plateau and were expected to decline, boosting efforts to make unconventional resources competitive. The gas market was competitive and deregulated, relying on a dense network of pipelines, a feature that reduced the risk of downstream bottlenecks for successful innovative entrants. Federal and State regulations were also relatively favorable, in particular reducing investors' responsibility over potential damages on water resources, and protecting secrecy of firm's specific hydraulic fracturing technology. These features combined with the institutional and social context. Oil and gas production is customary in much of the production sites, reducing local political opposition to drilling activity. From the institutional point of view, some States had very liberal regulation of oil and gas production and related regulation of industrial and environmental hazards (see Krupnick et al. (2015)).
} 
shale oil has also been dramatic and unexpected." According to Baumeister and Kilian (2016b) over half of the predictable drop in oil prices in the 3rd and 4th quarter of 2014 is attributable to positive supply shocks, which include information on the ongoing expansion of shale oil production in the U.S. Beyond shale oil, it is noteworthy that oil prices have been regularly subject to unexpected shocks as explained by Baumeister and Kilian (2016a). This feature, common to non-renewable resources markets, including minerals, is closely scrutinized in this article.

The Regulation of PRoduction. Mineral rights in the U.S. are attached to surface property, which may be private or public. Lease contracts have developed for oil and gas exploration and production. They include an upfront payment from the oil and gas company to the land owner, a deadline for beginning production, and royalty payments to the land owner proportional to production or value of sales. A part for the deadline for beginning production, the oil and gas company chooses the quantity to be produced at each date. The management of the resource is de facto delegated from the resource owner to the oil and gas company for the duration of the lease. Similar contracts rule the exploration and production over federal lands. State regulations apply to oil and gas activity, on top of Federal and State regulation on industrial and environmental hazards.

The shale oil and gas activity is spreading to other countries, including the Canada, Argentina, China and the U.K. In several cases foreign companies are involved in exploration and production programs. These firms are often large multinational corporations (hereafter IOC for International Oil Companies). In most cases, mineral rights are exclusively in the hand of public authorities, governments are involved in agreements with IOC over the activity. Historically, concession contracts were first used, transposing abroad the American contractual relationships whereby the IOC has to pay an upfront fee to become the residual claimant over the production. Governments seeking to improve their bargaining power developed and increasingly adopted an alternative form of contract, namely production sharing agreements (PSA). Under a PSA the resource owner keeps all the rights on the resource, which formally allows it to choose the quantity produced at each date. The government accepts to hand a share of production to the IOC, to compensate it for the cost of exploration and development (cost-oil) over the first years of production, and then a smaller share of production to grant some profitability of the operation to the IOC (profit-oil). ${ }^{2}$ Notice that despite the formal differences between concession contracts and PSAs, in practice most governments take part to the choices concerning the scale and rhythm of production also under concession contracts, either because their national oil company are directly involved or because contractual clauses include government approval of production plans.

When it comes to the assessment of the amount of resource in a given field, the IOC or the oil and gas company certainly benefit from superior information and knowledge with respect to governments or private landowners. As Osmundsen (2014) argues, the superior expertise of IOC as well as the complexity of petroleum operations open the door to the strategic reporting of costs or reservoir sizes at the different stages of the life cycle of the petroleum field (such as resource estimates at the licensing stage and cost reports at the production stage). Therefore, the contract established between the IOC and the

\footnotetext{
${ }^{2}$ Scholars distinguish a third type of contract, less common, that corresponds to a delayed fixed payment from the government to the IOC for exploration and development, possibly for the production operations, called service contract.
} 
resource owner should take into account the incentives of the firm to report strategically its information in its best interest.

MODEL AND MAIN RESUlTs. The questions we address can thus be stated as follows: First, how does asymmetric information shape the contractual relationship between the public authority, which owns the resource, and the IOC which is in charge of its production? Second, how does a structural break in the price of the resource affect the optimal extraction path and how should the contract take into account such a break?

To tackle those issues, we consider a model of regulation in which a government delegates to a firm the extraction of non-renewable resource through a contract. We consider that the initial stock of the resource is private information of the firm and affects the marginal cost of extraction: the higher the stock of resource, the lower is the marginal cost of extraction. We bear a particular attention on how the quantity of resource extracted varies over time. In practice, the contract concerns a concession area (a so-called "block"), over which the IOC can decide at which speed to develop new wells, if any. This makes it possible for the firm to manage production over time. As compared to the conventional oil and gas production, shale gas is characterized by greater flexibility. ${ }^{3}$

The price of the resource is assumed to follow an exogenous dynamics. This captures the fact that the resource is sold on a world market and that the firm has no influence on the world price, as it is the case for the oil and most mineral markets. To begin with, we consider a "smooth raising price dynamics" according to which, loosely speaking, the world price is increasing smoothly over time, traducing the progressive scarcity of the resource at the world level.

Our framework allows us to obtain a clear benchmark for the case of complete information. The first best extraction path has two interesting features. First, independently of the size of the reserve, the firm is asked to follow a unique extraction path, and to sell at the beginning of the contract the quantity of resource in excess of the stock that is worth selling later in light of the anticipated price path. Second, extraction always ends up in finite time: as price increases over time, the firm extracts less and less resource up to the point where the reserve is fully exhausted. We also show that a possible implementation of the optimal regulation under complete information consists in making the firm pay a bond ex ante and in leaving the firm with the discretion to choose the extraction path. This is tantamount to a concession contract with no royalty and the maximum license fee acceptable by the IOC.

When the firm possesses private information, the extraction path must be designed in a way that is compatible with the firm's incentives to manipulate its information. The delegated management of resource extraction should share some of the general features

\footnotetext{
${ }^{3}$ Abstracting the stochastic nature of the activity, the relative flexibility in the production schedule is due to the following features: first, for a given potential productivity of the shale play, production increases proportionally to the number and length of horizontal fracking wells; second, each single well produces most of the output over the first 24 months; third, reserves are embedded in shale plays that extend over very large areas. Hence, a firm that wishes to scale upward (downward) its production, can durably drill more or longer (less or shorter) wells that what would be required to replace declining production from aging wells.
} 
found with other forms of procurement. ${ }^{4}$ The literature has highlighted that the design of optimal contracts under informational constraints results from a trade-off between efficiency and rent extraction. ${ }^{5}$ Choosing to operate at the efficient level might also necessitate to leave excessive information rents, which might be viewed as socially costly by public authorities. Reducing information rents instead calls for lower output, lowpowered incentives and more generally procurement policies that are less sensitive to information.

We use the analysis undertaken in Martimort et al. (2018) to analyze the requirement of incentive compatibility on the extraction path. As in that paper, we assume that the government can commit to a contract. In line with our companion paper, we show that the Hotelling rule has to be modified and becomes a virtual Hotelling rule where the marginal cost of extraction is replaced by the virtual marginal cost. Hence, because the marginal costs of extraction differ now across fields with different reserves, the extraction paths as well as the termination dates of those fields differ as well.

We then introduce downward "structural breaks" in the price dynamics. Such durable reduction of the price characterize natural resource markets and result from demand shocks, such as the economic slowdown in the aftermath of the financial crisis, or from supply shocks following, for instance, the discovery of new reserves or of more efficient extraction technologies. We consider that the price jumps downwards and then follows the same dynamics as before the break. When considering price breaks, one issue concerns the capacity to anticipate those breaks. When a price break can be fully anticipated by the regulator and the firm, then the optimal extraction path is such that production must be stopped before the break. How long production is stopped depends only on the characteristics of the price break and not on the extraction cost. Therefore, a similar pattern emerges under incomplete information.

The analysis concludes with the perhaps more convincing case where the price break cannot be perfectly anticipated by the parties. This has consequences both on the extraction path and the design of the contract. Under complete information, and quite intuitively, granting ownership of the resource to the firm with the first best license fee is no longer feasible, for the firm would run a loss if it had paid an upfront price to become the owner which does not take into account the possibility of a price break. A better alternative is for the principal to keep ownership of the field but use a contract where at any date the firm keeps all control rights on production but pays a rental price equal to the current profit. This contract is first clearly immune to the possibility of an unexpected break since it does not require any upfront payment that eventually could not be covered ex post with realized profits in sharp contrast with the ownership solution. Second, with this scheme, the firm always makes the right production decision even following an unexpected price break. Such a contract resembles to a PSA, where costs are observable. Under symmetric information, PSAs allow to insure a minimum return on investment to the firm, avoiding to leave any extra surplus.

Under asymmetric information, PSAs no longer fare as well as under complete information. Indeed, the principal cannot extract the realized profit at each given date since

\footnotetext{
${ }^{4}$ See Laffont and Tirole (1993) and Armstrong and Sappington (2007) for accounts of the so-called New Regulatory Economics.

${ }^{5}$ Laffont and Martimort (2009).
} 
the cost function of the firm is private information ex ante and thus cannot be specified into a contract. Here, we should throw a word of caution by noticing that, although the firm's stock is unknown ex ante, the earlier path of extraction has already revealed this parameter. Ex post, when the unexpected break arises the cost function is thus perfectly known by all parties. Yet, an arrangement where those costs are compensated by the principal cannot be written ex ante and thus cannot be enforced.

Literature REVIEW. Our paper belongs to the literature on resource extraction and incentives. Osmundsen (1998), Poudou and Thomas (2000) and Hung et al. (2006) have analyzed the design of a mining concession contract in a (finite) multi-period adverse selection setting with a finite number of periods and a persistent shock on cost which is private information of the concessionaire. Reducing information rents requires downward distortions of production early in the relationship. With a finite number of periods, this implies that more resource can be extracted in a terminal phase of extraction. In a two-period model, Gaudet et al. (1995) studied optimal royalty contracts for a nonrenewable resource under asymmetric cost information with intertemporally independent cost parameters, and where the government cannot commit. ${ }^{6}$ A common finding of these papers is that asymmetric information shifts production to the future. We differ from these papers along several lines. First, our model has continuous time and infinite horizon in order not to constrain the resource patterns. This means that all effects that stem from the existence of a terminal date disappear in our context. Second, we assume that the cost of extraction depends both on current production and on the remaining stock as in Solow and Wan (1976), Pindyck (1978) and Pindyck (1987), Swierzbinski and Mendelsohn (1989).

Our analysis builds on a companion paper (Martimort et al. (2018)), which provides a theoretical framework addressing the question of the optimal regulation of a nonrenewable resource. There, we develop a framework where a regulator contracts with a firm for the extraction of a resource. Key to our analysis is the presence of asymmetric information between the regulator and the firm. We depart from that analysis in several important ways. First, the price of the resource, and its dynamics, are taken as exogenous. Second, we analyze the impact of structural breaks on the regulation.

\section{THE MODEL}

Resource Dynamics. We consider the dynamic management by a firm of a stock $S(\theta, t)$ of resources over time and how this management can be optimally regulated through a concession contract designed by a public authority (referred to as the principal in the sequel). The initial stock of resource at date $t=0$ is

$$
S(\theta, 0)=\theta
$$

The resource stock evolves according to the following differential equation

$$
\frac{\partial S}{\partial t}(\theta, t)=-q(\theta, t)
$$

\footnotetext{
${ }^{6}$ Castonguay and Lasserre (2016) extend the latter analysis to consider a preliminary stage where reserves are developed.
} 
where $q(\theta, t)$ denotes the non-negative amount of resources extracted at date $t$ starting from an initial stock $\theta$. For the rest of the analysis, it turns out that making this nonnegativity assumption explicit turns out to be useful

$$
q(\theta, t) \geq 0
$$

Production technology and preferences. Following the existing literature, we assume that extracting resources is all the more difficult that the remaining stock is low. Formally, we write the cost of extracting $q$ units when the remaining stock is $S$ as

$$
C(S) q
$$

Therefore, we assume constant returns-to-scale in extraction but we also posit that the marginal cost of production $C(S)$ is decreasing and convex in the level of that stock $\left(C^{\prime}<0 \leq C^{\prime \prime}\right)$. For technical reasons, we assume also that this marginal cost remains finite, $C(0)<+\infty$ and that $C^{\prime \prime \prime} \leq 0$.

The revenue from exploiting $q$ units of resource at date $t$ is denoted by $R(q, t)=p(t) q$, where $p(t)$ is the world price of resource which is fixed on a world market and thus taken as given for the rest of our analysis. The dynamics of the price is here taken as exogenous. ${ }^{7}$ In the sequel, it will be particularly useful to define the net present value $u(t)$ of one unit of resource at date $t$ as

$$
u(t)=p(t) \exp (-r t)
$$

where $r$ is the interest rate. We will assume that $u(t)$ is once differentiable and decreasing almost everywhere for most of this presentation, although later on, we will give specific attention to the case of a "break" in the price dynamics with the possibility of a discontinuous downward jump in the dynamics of $u(t)$ (see the details below for a definition). That $u(t)$ decreases captures the idea that, over time, suppliers may have invested in alternative technologies and thus other sources of resources may become available for consumption, reducing de facto the value of the resource under scrutiny.

As we will see below, some of the properties of optimal extraction paths depend of course on the properties imposed on $u(t)$ which are themselves inherited from the price dynamics. To get later on a sharp writing of the Hotelling rule, we also define the function $z(t)$ as

$$
z(t)=-\dot{u}(t) \exp (r t)
$$

Observe that $z(t)$ is positive whenever $u(t)$ is decreasing.

AsYMMETRIC INFORMATION. We will be particularly interested in the sequel in the scenario where the initial stock of resource $\theta$ may be private information to the firm. We then suppose that this parameter is drawn from the set $\Theta=[\underline{\theta}, \bar{\theta}]$ according to the cumulative (atomless) distribution $F(\cdot)$ with (strictly positive) density $f(\cdot)=F^{\prime}(\cdot)$.

Following the screening literature, we also assume that the so called Monotonicity of

\footnotetext{
${ }^{7}$ In our companion paper (Martimort et al. (2018)), we fully endogenize those price dynamics.
} 
the Hazard Rate Property holds: ${ }^{8}$

$$
\frac{d}{d \theta}\left(\frac{1-F(\theta)}{f(\theta)}\right) \leq 0 \text { for all } \theta \in \Theta
$$

That the firm has better information on the stock of resources than the regulator is in line with casual evidences that operators have more precise signals than outsiders (regulators, financiers, local communities) on the value of reserves.

\section{Smooth Raising Price Dynamics}

We start with the simple case where the price dynamics is such that $z(t)$ is continuously increasing over time. We first analyze the case of complete information before moving to the case where the firm holds private information on the initial level of resources it may extract. Beforehand, we propose the following definition.

DEFINITION 1. A smooth raising price dynamics is such that $z(t)$ is continuous and everywhere increasing.

\subsection{Complete Information}

When $\theta$ is common knowledge, the principal can let the firm enjoy all revenues from exploiting the resource and then fully extract the firm's profits through a lump-sum upfront payment equal to the discounted value of those profits. The only important question is to determine the optimal path of resource extraction. The next proposition describes carefully the optimal dynamics of extraction. We denote respectively by $q^{*}(\theta, t)$ and $S^{*}(\theta, t)$ the optimal quantity extracted at a time $t$ and the optimal remaining stock of resources at that date. This optimal path maximizes the intertemporal discounted profit subject to the constraints imposed by resource dynamics, that is

$$
\begin{aligned}
\left(\mathcal{P}^{*}(\theta)\right): & \max _{q, S, T} \int_{0}^{T}(p(t)-C(S(\theta, t))) q(\theta, t) \exp (-r t) d t \\
& \text { subject to }(2.1),(2.2) \text { and }(2.3) .
\end{aligned}
$$

The maximand is taken on the resource trajectories with an extra optimization variable being a priori the end-point date $T$ at which extraction ceases. In the above constrained set, we make explicit the non-negativity constraint on the amount of resource extracted at any point in time. This solution is easily characterized if we further impose the following assumption:

Assumption 1.

$$
z(0)>r C(\underline{\theta}) \text { and } \lim _{t \rightarrow+\infty} z(t)<r C(0)
$$

As the analysis below will demonstrate, the condition $z(0)>r C(\underline{\theta})$ ensures that extraction is valuable at date $t=0$ even for the lowest possible level of initial stock. Because $C(S)$ is decreasing, it follows that $z(0) \geq r C(\theta)$ for all $\theta \in \Theta$. The second inequality guarantees that complete extraction takes place in finite time.

\footnotetext{
${ }^{8}$ Bagnoli and Bergstrom (2005).
} 
Proposition 1. Suppose that Assumption 1 holds and consider a smooth raising price dynamics (i.e., $\dot{z}(t)>0$ for all $t$ ). The optimal path of resource extraction under complete information $\left(S^{*}(t), q^{*}(t)\right)$ follows the same standard Hotelling Rule whatever the initial level of resource $\theta$. This path is defined as:

$$
r C\left(S^{*}(t)\right)=z(t) \quad \forall t \in\left(0, T^{*}\right)
$$

and

$$
q^{*}(t)=-\frac{\dot{z}(t)}{r C^{\prime}\left(S^{*}(t)\right)}>0 \quad \forall t \in\left(0, T^{*}\right)
$$

where $T^{*}$, which is the finite date at which all fields are fully extracted, is defined as

$$
r C(0)=z\left(T^{*}\right) .
$$

This proposition showcases two important features. First, Assumption 1 ensures that at date $t=0$, all fields start extraction and do so maybe with a large output extraction at that date so that, right after that date, all fields jump on the same path of extraction $\left(S^{*}(t), q^{*}(t)\right)$. That all trajectories follow the same path of extraction even though they may start at different levels of resource at date $t=0$ is rather intuitive. After some initial period of extraction moving an initial stock $\theta$ to a lower value $\theta^{\prime}$, the continuation path must obviously be the same as if the stock had started from this lower value. This just follows from the Bellman Principle for optimization of a time-consistent objective.

This common path follows the usual Hotelling rule. Indeed, at the optimum, the firm (or alternatively the principal, remember that the former can be made residual claimant by the latter) must be just indifferent at any date $t$ between consuming some extra amount $d q$ today or delaying for an extra length of time that resource in the ground to facilitate extraction later on. Producing and consuming $d q$ more units beyond $q^{*}(t)$ for a length of time $d t$ around date $t$ increases overall profit at date $t$ by approximately

$$
\left(p(t)-C\left(S^{*}(t)\right)\right) d q d t .
$$

However, consuming those extra units $d q$ reduces the stock of resources from date $t+d t$ onward to approximately $S^{*}(t)-q^{*}(t) d t+d q d t$. Future costs of extraction are thus increased from date $t+d t$ onwards by an amount whose discounted value is worth approximately

$$
-\left(\int_{t}^{+\infty} C^{\prime}\left(S^{*}(\tau)\right) q^{*}(\tau) \exp (-r(\tau-t)) d \tau\right) d q d t
$$

At the optimal path, (3.4) and (3.5) must be equal so as to leave the firm indifferent between extracting that extra unit or not. This indifference can be written as:

$$
p(t)-C\left(S^{*}(t)\right)=-\left(\int_{t}^{+\infty} C^{\prime}\left(S^{*}(\tau)\right) q^{*}(\tau) \exp (-r(\tau-t)) d \tau\right) .
$$

Differentiating with respect to $t$ and simplifying finally gives us the Hotelling Rule (3.1).

This rule can be reinterpreted as viewing $z(t) / r$ as the current discounted value of extracting one more unit of output at date $t$. Condition (3.1) thus equals the marginal 
benefit of extracting one more unit with its costs.

The second important feature of the solution is that extraction always ends up in finite time when the firm faces a smooth raising price dynamics. As price increases (more precisely, as $z(t)$ increases) over time, the firm extracts less and less of the resource up to the point of exhausting all reserves in finite time $T^{*}$.

IMPLEMENTATION. As suggested above, a possible implementation of the optimal contract under complete information consists in leaving all revenues to the firm and extract a lump-sum payment equal to the net present value of its profits. Being residual claimant for optimal resource management, the firm chooses the optimal extracting path. This contractual solution is akin to leaving full ownership to the contractor at a type-dependent price $P^{*}(\theta)$ such that:

$$
P^{*}(\theta) \equiv \int_{0}^{T^{*}}\left(p(t)-C\left(\theta-\int_{0^{+}}^{t} q^{*}(\tau) d \tau\right)\right) q^{*}(t) \exp (-r t) d t .
$$

\subsection{Asymmetric Information}

We now turn to the case of asymmetric information, still assuming that the price dynamics remains smooth. The analysis in this section borrows from our companion paper Martimort et al. (2018) and we refer the reader to that paper for details of the analysis.

InCENTIVE COMPATIBILITY. A long-term regulatory contract is written so as to regulate the relationship between the public authority and the firm. This contract stipulates how much quantity should be extracted and possibly a payment for such service at any point in time. From the Revelation Principle, ${ }^{9}$ we can without loss of generality view such contract as a direct and truthful revelation mechanism that stipulates the output $q(\hat{\theta}, t)$ and the payment $\omega(\hat{\theta}, t)$ profiles over time as a function of the firm's announcement $\hat{\theta}$ on the resource stock it starts with. Because we assume that the principal commits to the mechanism, this announcement takes place once for all at date $0^{-} .{ }^{10}$

Henceforth, we may denote by $U(\theta)$ the firm's informational rent (or intertemporal payoff) when adopting such optimal truthful announcement strategy as

$$
U(\theta)=\int_{0}^{+\infty}(\omega(\theta, t)-C(S(\theta, t)) q(\theta, t)) \exp (-r t) d t
$$

where the trajectory $S(\theta, t)$ is defined through (2.1) and (2.2).

Incentive compatibility requires that this payoff is greater than what the firm with type $\theta$ may obtain by adopting the extraction patterns of a type $\hat{\theta}$ while still starting from the initial stock $\theta$. Incentive compatibility can thus be written as

$$
U(\theta)=\max _{\hat{\theta} \in \Theta} \int_{0}^{+\infty}\left(\omega(\hat{\theta}, t)-C\left(\theta-\int_{0}^{t} q(\hat{\theta}, \tau) d \tau\right) q(\hat{\theta}, t)\right) \exp (-r t) d t .
$$

\footnotetext{
${ }^{9}$ Myerson (1982).

${ }^{10}$ Observe also that there is no loss of generality in making the convention of an infinite planning horizon for the firm because the contract can just stipulates zero output and zero payment from a given date on if extraction takes place over a finite period of time.
} 
A contract $\left((\omega(\hat{\theta}, t), q(\hat{\theta}, t))_{t \geq 0}\right)_{\hat{\theta} \in \Theta}$ thus induces an allocation $\left(U(\theta),(q(\theta, t))_{t \geq 0}\right)_{\theta \in \Theta}$. Next Lemma, which is directly taken from our companion paper Martimort et al. (2018), characterizes incentive compatible allocations, i.e., allocations that can be implemented by a particular contract. We postpone below the analysis of contracts that implement the optimal allocation.

Lemma 1. An allocation $\left(U(\theta),(q(\theta, t))_{t \geq 0}\right)_{\theta \in \Theta}$ is incentive compatible if and only if:

1. $U(\theta)$ is absolutely continuous and thus a.e. differentiable with at any point of differentiability $\theta$ :

$$
\left.\dot{U}(\theta)=-\int_{0}^{+\infty} C^{\prime}(S(\theta, t)) q(\theta, t)\right) \exp (-r t) d t
$$

2. The amounts extracted satisfies the following conditions

$$
\int_{0}^{+\infty}\left(\int_{\theta}^{\hat{\theta}}\left(\int_{Q(\hat{\theta}, t)}^{Q(\tilde{\theta}, t)} C^{\prime}(\tilde{\theta}-\tilde{Q}) d \tilde{Q}\right) d \tilde{\theta}\right) \exp (-r t) d t \geq 0 \quad \forall(\theta, \hat{\theta}) \in \Theta^{2}
$$

In particular, (3.9) holds if the following sufficient condition is satisfied

$$
Q(\theta, t) \text { non-decreasing in } \theta \text {. }
$$

Proof. See Martimort et al. (2018).

The Envelope Condition (3.8), a standard condition for incentive compatibility repeatedly found in the mechanism design literature ${ }^{11}$ shows that the information rent of the firm is non-decreasing in its initial stock of resources. Hence, any type of firm accepts the contract if the firm with the lowest reserve $\underline{\theta}$ already does so. We will thus impose on top of the incentive compatibility conditions (3.8) and (3.10) the following participation constraint

$$
U(\underline{\theta}) \geq 0
$$

Together incentive and participation constraints define the incentive-feasible set.

Virtual Hotelling Rule. The principal's objective is to maximize his expected intertemporal payoff defined as

$$
\mathbb{E}_{\theta}\left(\int_{0}^{+\infty}(p(t) q(\theta, t)-\omega(\theta, t)) \exp (-r t) d t\right)
$$

subject to the equations (2.1), (2.2) and (2.3) that describe how the stock of resource evolves on path and subject to the firm's incentive and participation constraints (3.8), (3.9) and (3.11).

Taken together (3.8) and (3.11) (which is clearly binding at the optimum since the principal wants to reduce as much as possible the firm's payments) allows us to rewrite

\footnotetext{
${ }^{11}$ Laffont and Martimort (2009).
} 
a firm $\theta$ 's information rent as

$$
\begin{aligned}
U(\theta) & \left.=-\int_{\underline{\theta}}^{\theta}\left(\int_{0}^{+\infty} C^{\prime}(S(\tilde{\theta}, t)) q(\tilde{\theta}, t)\right) \exp (-r t) d t\right) d \tilde{\theta} \\
& =\int_{0}^{+\infty}\left(\omega(\theta, t)-C\left(\theta-\int_{0}^{t} q(\theta, \tau) d \tau\right) q(\theta, t)\right) \exp (-r t) d t .
\end{aligned}
$$

From this, we may derive the value of the discounted payment $\int_{0}^{+\infty} \omega(\theta, t) \exp (-r t) d t$ made to the firm, and insert into the principal's objective function (3.12) to obtain the new expression of this objective as

$$
\mathbb{E}_{\theta}\left(\int_{0}^{+\infty}(p(t) q(\theta, t)-C(S(\theta, t) q(\theta, t)) \exp (-r t) d t-U(\theta))\right.
$$

Further integrating by parts using (3.13) yields a last rewriting of this objective

$$
\mathbb{E}_{\theta}\left(\int_{0}^{+\infty}(p(t) q(\theta, t)-\tilde{C}(\theta, S(\theta, t) q(\theta, t)) \exp (-r t) d t)\right.
$$

The above maximand showcases that, under asymmetric information, the marginal cost of extraction is now replaced by the virtual marginal cost of extraction that is now defined as

$$
\tilde{C}(\theta, S)=C(S)-\frac{1-F(\theta)}{f(\theta)} C^{\prime}(S) \quad \forall(S, \theta)
$$

This expression accounts for the extra cost of information rent that has to be given to the firm to induce information revelation on the value of the stock. As a result, the virtual marginal cost of extraction is always above the true marginal cost of extraction

$$
\tilde{C}(\theta, S) \geq C(S) \quad \forall(S, \theta)
$$

with an equality only for the highest possible type $\bar{\theta}$.

The virtual marginal cost of extraction is increasing and convex in $S$ under the assumptions made earlier on for $C$. Importantly, the marginal cost of extraction can also be ranked with respect to the initial stock $\theta$ since

$$
\frac{\partial \tilde{C}}{\partial \theta}(\theta, S) \leq 0 \quad \forall(S, \theta) .
$$

A type with a higher initial stock has thus a lower virtual marginal cost of extraction and is more willing to extract, at any level of stock along the trajectory.

The analysis made in our companion paper Martimort et al. (2018) carries over in the present environment mutatis mutandis. In particular, the solution to this problem is obtained by solving separately a collection of maximization problems for each possible realization of $\theta$. Each of those problems can then be written respectively as

$$
\left(\mathcal{P}^{s b}(\theta)\right): \quad \max _{q, S, T} \int_{0}^{T}(p(t) q(\theta, t)-\tilde{C}(\theta, S(\theta, t)) q(\theta, t)) \exp (-r t) d t
$$




$$
\text { subject to (2.1), (2.2) and (2.3) . }
$$

The cost of extraction at any given date now depends not only on the current stock but also on the initial stock. Hence, firms become heterogeneous in terms of their costs of extraction while under symmetric information they all had the same technology and just differed by their initial stocks. Such heterogeneity will imply that the optimal paths of extraction under asymmetric information are now all different and depend on the initial stock of resources.

It is straightforward to extend mutatis mutandis the analysis undertaken under complete information to this new scenario. The next proposition does so, stressing that now firms with different initial stocks follow different paths of extraction. Before that, we impose a new assumption that is the counterpart of Assumption 1 under asymmetric information.

ASSUMPTiOn 2.

$$
z(0)>r \tilde{C}(\underline{\theta}, \underline{\theta}) \text { and } \lim _{t \rightarrow+\infty} z(t)<r \tilde{C}(\bar{\theta}, 0) .
$$

The monotonicity of the hazard rate (2.4) together with the monotonicity properties of $C$ and $C^{\prime}$ imply that $\tilde{C}(\theta, \theta)$ is a decreasing function of $\theta$. In other words, the first inequality implies that all firms, whatever their initial stock, start to produce and actually face a downward jump of their remaining stock at date $0^{+}$since $z(0)>r \tilde{C}(\theta, \theta)$ for all $\theta \in \Theta$. After this jump, the extraction path follows the smooth path described in Proposition 2 below. The second inequality also holds at all $\theta$ thanks again to the monotonicity properties of $C, C^{\prime}$ and $C^{\prime \prime}$. This condition implies that extraction always ends in finite time whatever the initial level of resource.

We are now ready to describe optimal extraction paths under asymmetric information.

Proposition 2. Suppose that Assumption 2 holds and consider a smooth raising price dynamics (i.e., $\dot{z}(t)>0$ for all $t$ ), the optimal path of resource extraction $\left(S^{s b}(\theta, t), q^{s b}(\theta, t)\right)$ under asymmetric information evolves according to a virtual Hotelling rule that depends on the initial level of resource $\theta$. This path is defined as follows:

$$
r \tilde{C}\left(\theta, S^{s b}(\theta, t)\right)=z(t) \quad \forall t \in\left(0, T^{*}(\theta)\right)
$$

and

$$
q^{s b}(\theta, t)=-\frac{\dot{z}(t)}{r \frac{\partial \tilde{C}}{\partial S}\left(\theta, S^{s b}(\theta, t)\right)}>0 \quad \forall t \in\left(0, T^{*}(\theta)\right)
$$

where $T^{*}(\theta)$, which is the finite date at which a field with initial reserves $\theta$ is fully extracted, is defined as

$$
r \tilde{C}(\theta, 0)=z\left(T^{*}(\theta)\right) .
$$

Proposition 2 is much alike Proposition 1. The difference is that the costs of extraction are now replaced by the virtual costs of extraction. Because those costs differ across fields with different reserves, the extraction paths of those fields differ as well.

For a given initial stock of resource, the dynamics looks quite similar to the case of complete information. To understand this effect, it is important to come back on 
(3.8). This formula shows that a type- $\theta$ firm enjoys some information rent by extracting the same amount as a firm with a lower initial stock but doing so at a lower marginal cost. This rent is reduced when the regulator calls for lower quantities. Asking for less extraction by a given type $\theta$ indeed reduces the information rent of all supramarginal types $\theta^{\prime} \geq \theta$. In other words, doing as if more of the resource had already been exploited by a given type and pushing forward the extraction path for that type helps to extract rent from types endowed with more resource. The second-best path of extraction exhibits a trade-off between implementing ambitious extraction paths that come close to efficiency and, to do so, leaving excessive information rent to the firm. Extracting more of this information rent calls for extracting the resource at a slower pace.

Next Lemma illustrates this insight by looking at the date at which different fields are exhausted.

Lemma 2. $T^{*}(\theta)$ is an increasing function of $\theta$ with $T(\bar{\theta})=T^{*}$.

While under complete information extraction ends at the same date for all fields, this is no longer the case under asymmetric information. Fields with larger initial stocks extract during longer periods.

IMPLEMENTATION. We now address the question of what sort of instruments can be used to achieve the second-best patterns highlighted in Proposition 2. In a dynamic environment, there are many possible ways to structure incentive payments over time to provide the needed information rent. In our companion paper Martimort et al. (2018), we discuss how a royalty scheme can be implemented based on the firm's prediction on the actual extraction path it will follow. In that implementation, the operator is offered a menu of options involving different royalties and license fees, among which it chooses right at the start of extraction. This choice commits the firm on an extraction path.

For the purpose of the analysis undertaken in Section 4.2 below, we now propose a simple dynamic implementation where choices are repeated over time although those choices do not convey more information to the principal, i.e., all the operator's private information ends up being already revealed right at the start of the extraction phase. Our procedure takes advantage of the dynamic structure of the firm's information rent. Indeed, observe that we can write the intertemporal rent of the firm as a stream of per-period rents $\tilde{u}(\theta, t)$

$$
U(\theta)=\int_{0}^{+\infty} \tilde{u}(\theta, t) \exp (-r t) d t
$$

where the type- $\theta$ rent at date $t \tilde{u}(\theta, t)$ is

$$
\tilde{u}(\theta, t)=-\int_{\underline{\theta}}^{\theta} C^{\prime}(S(\tilde{\theta}, t)) q(\tilde{\theta}, t) d \tilde{\theta} .
$$

This formulation suggests that we may implement those per-period rents with a sequence of nonlinear prices $(\Omega(Q, q, t))_{t \geq 0}$ that determines the firm's payment at date $t$ as a function of the past (observed) overall quantity that has been extracted $Q$, the current level of extraction $q$ and calendar time $t$. Formally, let define

$$
\hat{u}(\theta, Q, t)=\max _{q \geq 0} \Omega(Q, q, t)-C(\theta-Q) q .
$$


Assuming quasi-concavity in $q$ of the firm's objective, the optimal quantity $\hat{q}(\theta, Q, t)$ chosen at date $t$ by a firm with initial stock $\theta$ and having already extracted an overall quantity $Q$ can be written as

$$
\frac{\partial \Omega}{\partial q}(Q, \hat{q}(\theta, Q, t), t)=C(\theta-Q)
$$

Condition (3.17) can be rewritten, at any interior point on the optimal trajectory, as

$$
q^{s b}(\theta, t)=-\frac{\dot{z}(t)}{r\left(C^{\prime}\left(\theta-Q^{s b}(\theta, t)\right)-\frac{1-F(\theta)}{f(\theta)} C^{\prime \prime}\left(\theta-Q^{s b}(\theta, t)\right)\right)} \quad \forall t \in\left(0, T^{*}(\theta)\right)
$$

where $Q^{s b}(\theta, t)=\int_{0}^{t} q^{s b}(\theta, \tau) d \tau$ is the second-best profile of cumulative extraction. We generalize this condition off-path, i.e., for any cumulative levels of extraction $Q$ that could be different from $Q^{s b}(\theta, t)=\theta-S^{s b}(\theta, t)$ so as to get

$$
\hat{q}(\theta, Q, t)=-\frac{\dot{z}(t)}{r\left(C^{\prime}(\theta-Q)-\frac{1-F(\theta)}{f(\theta)} C^{\prime \prime}(\theta-Q)\right)} \quad \forall t \in\left(0, T^{*}(\theta)\right)
$$

Provided that $C^{\prime \prime \prime} \leq 0$ and thanks to the monotone hazard rate property, this condition can be inverted to get a function $\hat{\Theta}(q, Q, t)$ that associates to each possible level of current production $q$ and each possible level of cumulative extraction that the type- $\theta$ reaches at date $t$. Coming back on (3.21), we can thus write

$$
\frac{\partial \Omega}{\partial q}(Q, q, t)=C(\hat{\Theta}(q, Q, t)-Q) \quad \forall(q, Q, t)
$$

which can be integrated to get the shape of the nonlinear payment schedule $\Omega(Q, q, t)$ as

$$
\Omega(Q, q, t)=\int_{0}^{q} C(\hat{\Theta}(\tilde{q}, Q, t)-Q) d \tilde{q}+\Psi(Q, t) \quad \forall(Q, t)
$$

where $\Psi(Q, t)$ is some arbitrary function of $(Q, t)$ that is defined at the boundary condition by the zero-profit requirement for the $\underline{\theta}$ type, namely $\tilde{u}(\underline{\theta}, t)=0$ or

$\Psi\left(Q^{s b}(\underline{\theta}, t), t\right)=-\int_{0}^{q^{s b}(\underline{\theta}, t)} C\left(\hat{\Theta}\left(\tilde{q}, Q^{s b}(\underline{\theta}, t), t\right)-Q^{s b}(\underline{\theta}, t)\right) d \tilde{q}+C\left(\underline{\theta}-Q^{s b}(\underline{\theta}, t)\right) q^{s b}(\underline{\theta}, t)$.

A standard application of the Envelope Theorem plus the imposed requirement that $\hat{u}(\underline{\theta}, Q, t)=0$ gives us

$$
\hat{u}(\theta, Q, t)=-\int_{\underline{\theta}}^{\theta} C(\tilde{\theta}-Q) \hat{q}(\tilde{\theta}, Q, t) d \tilde{\theta} .
$$

We thus have

$$
\tilde{u}(\theta, t) \equiv \hat{u}(\theta, Q(\theta, t), t) .
$$

At any interior point on the optimal trajectory, the optimality condition (3.21) be- 
comes

$$
\frac{\partial \Omega}{\partial q}\left(Q^{s b}(\theta, t), q^{s b}(\theta, t), t\right)=C\left(S^{s b}(\theta, t)\right)=\frac{z(t)}{r}+\frac{1-F(\theta)}{f(\theta)} C^{\prime}\left(S^{s b}(\theta, t)\right)<\frac{z(t)}{r} .
$$

This formula shows that, at the margin, the firm chooses to lower current extraction below the complete information level as required by the optimal trajectory under asymmetric information. In other words, this implementation is akin to using a time-dependent royalty

$$
R(\theta, t)=-\frac{1-F(\theta)}{f(\theta)} C^{\prime}\left(S^{s b}(\theta, t)\right)
$$

which, for any given type $\theta$, increases over time independently of the resource price $z(t)$.

We end this paragraph by noticing that leaving ownership to the firm is no longer optimal. Although it allows an efficient extraction of resource and all types whatever their initial stock of resources choose to move to that path at date $0^{+}$, it also leaves too much rent to the firm. Indeed, the highest price that would be paid by the firm for getting ownership would be

$$
P^{*}(\underline{\theta}) \equiv \int_{0}^{T^{*}}\left(p(t)-C\left(\underline{\theta}-\int_{0^{+}}^{t} q^{*}(\tau) d \tau\right)\right) q^{*}(t) \exp (-r t) d t .
$$

Requesting such payment leaves extra benefits to a type- $\theta$ firm

$$
P^{*}(\underline{\theta})-P^{*}(\theta) \equiv \int_{0}^{T^{*}}\left(C\left(\underline{\theta}-\int_{0^{+}}^{t} q^{*}(\tau) d \tau\right)-C\left(\theta-\int_{0^{+}}^{t} q^{*}(\tau) d \tau\right)\right) q^{*}(t) \exp (-r t) d t .
$$

\section{PRICE BREAKS}

We now analyze how contracts should optimally be designed to react to the possibility of a price break. We have here in mind the possibility that the world market be modified by the discoveries of new sources of resources which change discontinuously how the value of one extra unit of extracted resource changes. To make such statement more formal, we propose the following definition

DEFINITION 2. A raising price dynamics exhibits a price break at date $\hat{T}$ if and only if there exists a smooth raising price dynamics $\tilde{z}(t)$ such that

$$
z(t)= \begin{cases}\tilde{z}(t) & \text { if } t \leq \hat{T} \\ \tilde{z}(t-\Delta) & \text { if } t>\hat{T}\end{cases}
$$

This definition of a price break bears on the properties of $z(t)$. It is quite natural since only $z(t)$ matters to characterize the Hotelling rule in our previous analysis. We require that $z(t)$, which represents how the net present value of extraction evolves over time, has a downward jump at some date $\hat{T}$

$$
\Delta z \equiv z(\hat{T})-z\left(\hat{T}^{+}\right)=z(\hat{T})-z(\hat{T}-\Delta) .
$$

We will assume in the sequel (and make this requirement implicit) that this jump is small enough so as to $z(t)$ remains everywhere positive and that $\hat{T}+\Delta<T^{*}$; the latter 
requirement will make it sure that the "no extraction area" (soon to be defined) ends before $T^{*}$. Beyond the break at $\hat{T}$, the net present value of extraction goes back to a dynamics that is similar to what was on course before the break but which is now delayed by $\Delta$.

This definition is actually consistent with different patterns of price dynamics. The price profile $p(t)$ might be a continuous function of $t$ with a discontinuous jump at $\hat{T}$; or only its derivative may have such jump. For technical reasons, we will favor the first possibility in the sequel, since the optimization problems remain then with continuous objectives, thereby avoiding the use of non-smooth calculus. ${ }^{12}$ In any case, and although the shape of the $z(t)$ profiles that we consider look specific, the analysis we develop below could be readily extended to more general patterns. The key assumption is that $z(t)$ is not everywhere increasing.

Implicit in our analysis of Section 3.1 and especially in the Hotelling rule (3.1) was the fact that when $z(t)$ is continuously strictly increasing, extraction is always positive at any point in time before exhaustion of the stock of resource. The possibility that $z(t)$ may decrease opens however the possibility that it becomes preferable to suspend extraction because the current value of doing so may fall short of the current stock.

Of course, the analysis changes depending on whether those breaks can be anticipated by parties to the contract or are just unexpected. We devote a separate section to each case.

\subsection{Expected Breaks}

Consider first the scenario where the break can be anticipated. Next proposition shows how parties should design the optimal extraction path under those circumstances.

Proposition 3. Suppose that Assumption 1 holds and consider a price dynamics with a break at $\hat{T}$. The optimal path of resource extraction under complete information $\left(S_{b}^{*}(t), q_{b}^{*}(t)\right)$ follows the same standard Hotelling Rule whatever the initial level of resource $\theta$. This path is defined as follows:

$$
\begin{gathered}
S_{b}^{*}(t)=S^{*}(t) \text { and } q_{b}^{*}(t)=q^{*}(t) \geq 0 \quad \forall t \in\left(0, t_{1}\right] \cup\left[t_{2}+\Delta, T^{*}\right) ; \\
S_{b}^{*}(t)=S_{0} \text { and } q_{b}^{*}(t)=0 \quad \forall t \in\left[t_{1}, t_{2}\right]
\end{gathered}
$$

where

$$
\begin{gathered}
r C\left(S^{*}(t)\right)=z(t) \quad \forall t \in\left(0, t_{1}\right] \cup\left[t_{2}+\Delta, T^{*}\right) ; \\
S_{0}=S^{*}\left(t_{1}\right)=S^{*}\left(t_{2}\right) ; \\
t_{2}-t_{1}=\Delta ;
\end{gathered}
$$

\footnotetext{
${ }^{12}$ It would be straightforward to extend the analysis to this case with just a little bit of extra burden; see Clarke (1990) for instance.
} 
and $t_{1}<\hat{T}$ solves

$$
u\left(t_{1}+\Delta\right)=u\left(t_{1}\right)+\dot{u}\left(t_{1}\right) \frac{1-\exp (-r \Delta)}{r} .
$$

The final date $T_{b}^{*}=T^{*}+\Delta>\hat{T}$ at which extraction stops solves:

$$
r C(0)=z\left(T_{b}^{*}\right)
$$

This proposition characterizes how the possibility of a price break can be managed when it is fully anticipated. The firm should stop production at a date $t_{1}$ that arises before the value of current extraction starts declining at $\hat{T}$ so as to start again production sooner in the future after a length of time worth $\Delta$. Of course, in practice, it may be hard to disrupt production at all and the insights provided here is only indicative that production should be carefully slowed down.

What is remarkable is that the bunching area $\left[t_{1}, t_{2}\right]$ only depends of characteristics of the price dynamics. Costs play no role whatsoever. The immediate consequence of this finding is that the same pattern is found also under asymmetric information.

Proposition 4. Suppose that Assumption 2 holds and consider a price dynamics with a break at $\hat{T}$. The optimal path of resource extraction under asymmetric information $\left(S_{b}^{s b}(\theta, t), q_{b}^{s b}(\theta, t)\right)$ follows a Virtual Hotelling Rule that depends on the initial level of stock $\theta$. This path is defined as follows:

$$
\begin{array}{cc}
S_{b}^{s b}(\theta, t)=S_{0}^{s b}(\theta) \text { and } q_{b}^{s b}(\theta, t)=q^{s b}(\theta, t) \geq 0 & \forall t \in\left(0, t_{1}\right] \cup\left[t_{2}+\Delta, T^{*}\right) ; \\
S_{b}^{s b}(\theta, t)=S_{0}^{s b}(\theta) \text { and } q_{b}^{s b}(\theta, t)=0 \quad \forall t \in\left[t_{1}, t_{2}\right]
\end{array}
$$

where

$$
S_{0}^{s b}(\theta)=S^{s b}\left(\theta, t_{1}\right)=S^{s b}\left(\theta, t_{2}\right)
$$

the boundaries of the no-production area $t_{2}$ and $t_{1}$ satisfy (A.17) and (4.7) and the final date of extraction $T_{b}^{*}(\theta)$ is equal to $T^{*}(\theta)+\Delta$ where $T^{*}(\theta)$ still satisfies (3.18).

The remarkable finding is that all types of firm, whatever the level of their initial stocks, stop extracting at the same date and for the same duration anticipating the price break; although, and in sharp contrast with complete information, the level of remaining stocks $S_{0}^{s b}(\theta)$ at which they do so differ.

\subsection{Unexpected Price Breaks and the Costs and Benefits of Concession Contracts}

The possibility of a price break might be hard to anticipate for the parties. This has consequences both on the extraction path and on contract design.

Complete Information. Consider first the case of complete information. Reaching date $\hat{T}$, the value of extracting one more unit of output $z\left(\hat{T}^{+}\right) / r$ discontinuously falls below its cost $C\left(S^{*}(\hat{T})\right)$ and thus extraction should cease, waiting for a duration $\Delta$ before which $z(\hat{T}+\Delta)$ reaches again the value $z\left(\hat{T}^{-}\right)$so as to start extraction again. 
The important point to notice is what sort of contracts or ownership structure allows to reach this outcome. Clearly, granting ownership to the firm is no longer feasible. Indeed, if the firm has paid upfront a price $P^{*}(\theta)$ to become an owner it would run a loss once the price break arises since from date $\hat{T}$ on the price becomes so low that production is no longer optimal and the optimal production plan is to depart from the (wrongly) expected output $\tilde{q}^{*}(t)$. Instead of reaching an expected profit

$$
\int_{\hat{T}}^{+\infty}\left(\tilde{p}(t)-C\left(\theta-\int_{0^{+}}^{t} \tilde{q}^{*}(\tau) d \tau\right)\right) \tilde{q}^{*}(t) \exp (-r t) d t
$$

where $\tilde{p}(t)$ and $\tilde{q}^{*}(t)$ are respectively the price and the optimal quantity associated to the (wrongly) expected dynamics $\tilde{z}(t)$, the firm gets only the lower profit

$$
\int_{\hat{T}+\Delta}^{+\infty}\left(\tilde{p}(t-\Delta)-C\left(\theta-\int_{0^{+}}^{t} q_{b}^{*}(\tau-\Delta) d \tau\right)\right) q_{b}^{*}(t) \exp (-r t) d t
$$

from date $\hat{T}$ on.

A better alternative for the principal is thus to keep ownership of the field but use a concession contracts where the firm enjoys all revenues from managing the resource. This solution also leaves to the firm all control rights on production, but it requests, at any possible date, a rental price equal to the current profit

$$
(p(t)-C(\theta-Q(t))) q(t)
$$

whatever the current extraction $q(t)$ and the cumulative past extraction $Q(t)$. This scheme is first clearly immune to the possibility of an unexpected break since it does not require any upfront payment that eventually could not be covered ex post with realized profits in sharp contrast with the ownership solution. Second, with this scheme, the firm always makes the right production decision even following an unexpected price break. Such a regulation corresponds to a PSA whereby the principal reimburses the cost, and leaves the responsibility of managing production to the firm. The contract can be signed ex ante, since the cost is known under full information.

Asymmetric Information. Asymmetric information makes things much more difficult. First, relinquishing control rights on production either through a concession contract or through ownership to the firm still allows a better adaptation of decisions to the unexpected price break. The firm still chooses to eschew production when its current return is too low. Unfortunately, at any point in time, the firm would be producing too much with those arrangements compared to the optimal second-best solution that, as we saw earlier, slows down extraction. Having the firm choosing freely its output ex post is bad from a rent extraction point of view. Second, granting ownership to the firm raises the same difficulty as under complete information, i.e., the price paid upfront would not be recouped with future profits if some unexpected break were to arise. Hence, firm's ownership remains quite unattractive.

Concession contracts have their own curse as well and they no longer fare as well as under complete information. Indeed, and in sharp contrast with the case of complete information, the principal cannot commit to a rental price equal to the realized profit at each given date since the cost function of the firm is private information ex ante and 
thus cannot be specified into a contract. Here, we should throw a word of caution by noticing that, although the firm's stock is unknown ex ante, the earlier path of extraction has already revealed this parameter. Ex post, when the unexpected break arises at date $\hat{T}$ the cost function is thus perfectly known by all parties. Yet, an arrangement where those costs are compensated by the principal cannot be written ex ante and thus cannot be enforced. Technically, everything happens as if the stock $\theta$ was observable ex post although not contractible ex ante.

One possibility consistent with the information structure is for the principal to ask at any point in time for

$$
(p(t)-C(\underline{\theta}-Q(t))) q(t)
$$

where the current extraction the cumulative past extraction are respectively $q(t)$ and $Q(t)$. Of course, this scheme fares badly in terms of extracting rent whenever $q(t)$ is chosen to be positive but it preserves the firm from being asked to pay when the price has fallen too far to warrant production.

\section{CONClusion}

We develop a model in which a firm extracts a resource, has private information about the initial level of resource, and is regulated. We characterize the optimal contract and extraction paths under two different scenarios about the dynamics of the resource price: first, when the resource price increases smoothly across time; second, when the resource price can be affected by a price break. We discuss how the anticipation of the price break, or the impossibility to anticipate it, affects the optimal extraction path. Our analysis could be further extended to better understand how various elements of organizational design, such as control rights in unforeseen contingencies, may be adequately used in these contexts.

\section{REFERENCES}

Armstrong, M. and Sappington, D. E. (2007). Recent developments in the theory of regulation. Handbook of industrial organization, 3:1557-1700.

Bagnoli, M. and Bergstrom, T. (2005). Log-concave probability and its applications. Economic theory, 26(2):445-469.

Baumeister, C. and Kilian, L. (2016a). Forty years of oil price fluctuations: Why the price of oil may still surprise us. Journal of Economic Perspectives, 30(1):139-60.

Baumeister, C. and Kilian, L. (2016b). Understanding the decline in the price of oil since june 2014. Journal of the Association of Environmental and Resource Economists, $3(1): 131-158$.

Castonguay, F. and Lasserre, P. (2016). Resource agency relationship with privately known exploration and extraction costs. Technical report, CIRANO.

Clarke, F. H. (1990). Optimization and nonsmooth analysis, volume 5. Siam. 
EIA (2015). Energy Information Administration: Annual Energy Outlook 2015 with projections to 2040. Technical report, US Energy Information Administration, Office of Integrated and International Energy Analysis, US Department of Energy, Washington, DC.

Gaudet, G., Lasserre, P., and Van Long, N. (1995). Optimal resource royalties with unknown and temporally independent extraction cost structures. International Economic Review, pages $715-749$.

Hung, N. M., Poudou, J.-C., and Thomas, L. (2006). Optimal resource extraction contract with adverse selection. Resources Policy, 31(2):78-85.

Joskow, P. (2015). The shale gas revolution: introduction. Economics of Energy \&6 Environmental Policy, 4(1).

Krupnick, A., Richardson, N., Gottlieb, M., et al. (2015). Heterogeneity of state shale gas regulations. Economics of Energy \& Environmental Policy, 4(1).

Laffont, J.-J. and Martimort, D. (2009). The theory of incentives: the principal-agent model. Princeton university press.

Laffont, J.-J. and Tirole, J. (1993). A theory of incentives in procurement and regulation. MIT press.

Martimort, D., Pouyet, J., and Ricci, F. (2018). Extracting information or resource? The Hotelling rule revisited under asymmetric information. The RAND Journal of Economics, 49(2).

Myerson, R. B. (1982). Optimal coordination mechanisms in generalized principal-agent problems. Journal of Mathematical Economics, 10(1):67-81.

Osmundsen, P. (1998). Dynamic Taxation of Non-renewable Natural Resources Under Asymmetric Information About Reserves. Canadian Journal of Economics, 31(4):933951.

Osmundsen, P. (2014). Time Consistency in Petroleum Taxation. The Taxation of Petroleum and Minerals: Principles, Problems, and Practice. FMI and Routledge.

Pindyck, R. S. (1978). The Optimal Exploration and Production of Nonrenewable Resources. Journal of Political Economy, 86(5):841-861.

Pindyck, R. S. (1987). On monopoly power in extractive resource markets. Journal of Environmental Economics and Management, 14(2):128-142.

Poudou, J.-C. and Thomas, L. (2000). Concession minière et asymétrie d'information. Économie et Prévision, 143(2):129-138.

Seierstad, A. and Sydsaeter, K. (1986). Optimal control theory with economic applications. Elsevier North-Holland.

Solow, R. M. and Wan, F. Y. (1976). Extraction Costs in the Theory of Exhaustible Resources. Bell Journal of Economics, 7(2):359-370. 
Swierzbinski, J. E. and Mendelsohn, R. (1989). Exploration and Exhaustible Resources: The Microfoundations of Aggregate Models. International Economic Review, $30(1): 175-186$.

\section{APPENDIX A}

Proof of Proposition 1. Problem $\left(\mathcal{P}^{*}(\theta)\right)$ is a maximization problem with free-end point to which we apply Pontryagin Principle:

$$
\begin{gathered}
\left(\mathcal{P}^{*}(\theta)\right): \max _{T, q, S} \int_{0}^{T}(p(t)-C(S(\theta, t)) q(\theta, t) \exp (-r t) d t \\
\text { subject to }(2.1) \text { and }(2.2) .
\end{gathered}
$$

We write the Hamiltonian for problem $\left(\mathcal{P}^{*}(\theta)\right)$ as:

$$
\mathcal{H}(S, q, t, \lambda)=(p(t)-C(S)) q \exp (-r t)-\lambda q
$$

where $\lambda$ is the costate variable for (2.2). This Hamiltonian is concave in $(q, S)$. The necessary conditions for optimality are thus also sufficient conditions of the Mangasarian type. ${ }^{13}$ We write those conditions as follows:

- Costate variable:

$$
\dot{\lambda}(t)=-\frac{\partial \mathcal{H}}{\partial S}\left(S^{*}(\theta, t), q^{*}(\theta, t), t, \lambda(t)\right)
$$

which amounts to

$$
\dot{\lambda}(t)=\exp (-r t) q^{*}(\theta, t) C^{\prime}\left(S^{*}(\theta, t)\right)
$$

- Control variable:

$$
q^{*}(\theta, t) \in \arg \max _{q} \mathcal{H}\left(S^{*}(\theta, t), q, t, \lambda(t)\right)
$$

which amounts to

$$
\exp (-r t)\left(p(t)-C\left(S^{*}(\theta, t)\right)\right)-\lambda(t) \leq 0 \text { with }=0 \text { if } q^{*}(\theta, t)>0 .
$$

- Free-end point and transversality condition:

1. If $T^{*}(\theta)<+\infty$, we should have:

$$
\lambda\left(T^{*}(\theta)\right)=0 \text { and }\left(p\left(T^{*}(\theta)\right)-C\left(S^{*}\left(\theta, T^{*}(\theta)\right)\right)\right) q^{*}\left(\theta, T^{*}(\theta)\right)=0 .
$$

2. If $T^{*}(\theta)=+\infty$, we should have:

$$
\lim _{t \rightarrow+\infty} \lambda(t)=0 \text { and } \lim _{t \rightarrow+\infty} \exp (-r t)\left(p(t)-C\left(S^{*}(\theta, t)\right)\right) q^{*}(\theta, t)=0 .
$$

Several facts follow from those optimality conditions.

Observe that differentiating (A.2) with respect to $t$ when $q^{*}(\theta, t)>0$ yields:

$$
\dot{\lambda}(t)+r \lambda(t)=\exp (-r t)\left(\dot{p}(t)+C^{\prime}\left(S^{*}(\theta, t)\right) q^{*}(\theta, t)\right) .
$$

\footnotetext{
${ }^{13}$ Seierstad and Sydsaeter (1986).
} 
Using (A.1) and (A.2) and simplifying yields:

$$
\dot{p}(t)=r\left(p(t)-C\left(S^{*}(\theta, t)\right)\right) .
$$

Now observe that

$$
\dot{p}(t)-r p(t)=\dot{u}(t) \exp (r t)=-z(t) .
$$

Hence, (A.6) can be rewritten as the Hotelling rule (3.1). This condition fully determine the common path $S^{*}(t)$ for all $t>0$ and all initial level $S^{*}(\theta, 0)=\theta$. At $t=0^{+}$, the optimal path jumps downward from $\theta$ to $S^{*}(0)$ which is lower from Assumption 1 and which is defined as:

$$
r C\left(S^{*}(0)\right)=z(0) .
$$

Differentiating with respect to $t$ for $t \in\left(0, T^{*}\right)$ gives us the common level of extraction $q^{*}(t)$ given by (3.2). For $t>T^{*}, S^{*}(t)=q^{*}(t)=0$. Finally, the final date of extraction $T^{*}=T^{*}(\theta)$ for all $\theta$ is such that $S^{*}(t)$ is positive on a left-neighborhood. It is of course given by (3.2).

Running EXAmple. Observe that we may rewrite (3.2)

$$
\begin{gathered}
S^{*}(t)=\frac{\frac{z(t)}{r}-C(0)}{C^{\prime}(0)}, \\
q^{*}(t)=\frac{\dot{z}(t)}{r C^{\prime}(0)} .
\end{gathered}
$$

where $T^{*}$ is defined by (3.3).

Proof of Lemma 1. See Martimort et al. (2018).

Proof of Lemma 2. Differentiating (3.18) with respect to $\theta$ gives:

$$
\dot{z}\left(T^{*}(\theta)\right) \dot{T}^{*}(\theta)=r \frac{\partial \tilde{C}}{\partial \theta}(\theta, 0)<0
$$

where the last inequality follows from (3.15). From this and the fact that $\dot{z}(t)>0$, we deduce that $\dot{T}^{*}(\theta)>0$.

Proof of Proposition 2. This is the almost same proof as for Proposition 1 mutatis mutandis provided that $C(S)$ and $S^{*}$ are respectively replaced by $\tilde{C}(\theta, S)$ and $\tilde{S}(\theta)$. Details are thus omitted. The only minor difference comes from Step 3 which has to be rewritten with some care. Indeed, for the optimal path of resource, we may again rewrite (2.2) as

$$
\frac{\partial S^{s b}}{\partial t}(\theta, t)=-q^{s b}(\theta, t)
$$

Replacing with $\tilde{C}(\theta, S)$, Equation (A.10) becomes:

$$
\frac{\partial q^{s b}}{\partial t}(\theta, t)=\frac{r}{V^{\prime \prime}\left(q^{s b}(\theta, t)\right)}\left(V^{\prime}\left(q^{s b}(\theta, t)\right)-\tilde{C}\left(\theta, S^{s b}(\theta, t)\right)\right) .
$$


The system made of (A.9) and (A.10) admits a limit, which is necessarily the unique stationary point $(\tilde{S}(\theta), 0)$. If convergence towards that point occurs in finite time $T^{s b}(\theta)<+\infty$, we demonstrate that output is necessarily null along the whole trajectory. Indeed, by CauchyLipschitz Theorem, there is a unique solution to the system now made of (A.9) and (A.10) with the initial condition $\left(S^{s b}\left(\theta, T^{s b}(\theta)\right), q^{s b}\left(\theta, T^{s b}(\theta)\right)\right)=(\tilde{S}(\theta), 0)$ and this is $\left(S^{s b}(\theta, t), q^{s b}(\theta, t)\right)=$ $(\tilde{S}(\theta), 0)$ itself. But this contradicts the initial condition $S^{s b}(\theta, 0)=\theta>\underline{\theta}$ if $\theta>\tilde{S}(\theta)$. Hence, the system cannot converge in finite time towards $(\tilde{S}(\theta), 0)$ and $q^{s b}(\theta, t)$ remains positive. Finally, gathering everything the system (A.9)-(A.10) converges towards $(\tilde{S}(\theta), 0)$ in infinite time when $\theta>\tilde{S}(\theta)$.

Suppose instead that $\theta \leq \tilde{S}(\theta)$, or equivalently (because $\tilde{C}(\theta, S)$ is decreasing in $S$ )

$$
\tilde{C}(\theta, \theta) \geq \tilde{C}(\theta, \tilde{S}(\theta))=P(0) .
$$

Then extraction is not even valuable for the first unit and zero extraction takes place for those lower values of $\theta$.

Proof of Proposition 3. We now make explicit the non-negativity constraint on output (2.3) and denote by $\mu$ the corresponding multiplier. We write the Hamiltonian and the Lagrangean for the corresponding optimization problem respectively as:

$$
\mathcal{H}(S, q, t, \lambda)=(p(t)-C(S)) q \exp (-r t)-\lambda q
$$

and

$$
\mathcal{L}(S, q, t, \lambda, \mu)=(p(t)-C(S)) q \exp (-r t)-\lambda q+\mu q .
$$

Proceeding as in the proof of Proposition 1, and looking for solutions such that $\mu(t)$ is continuously differentiable, we get the following condition:

$$
\dot{p}(t)+\dot{\mu}(t)=r\left(p(t)+\mu(t)-C\left(S_{b}^{*}(\theta, t)\right)\right)
$$

or

$$
z(t)-r C\left(S_{b}^{*}(\theta, t)\right)=\dot{\mu}(t)-r \mu(t) .
$$

We look for an interval $\left[t_{1}, t_{2}\right]$ where $(2.3)$ is binding. On the complementary set $\in\left(0, t^{*}\right] \cup\left[t^{*}+\right.$ $\left.\Delta, T^{*}\right)$, we have $\mu(t)=0$. The solution has the same features as in (4.2) and it does not depend on $\theta$ so that we omit this variable for brevity and write $S_{b}^{*}(\theta, t)=S_{b}^{*}(t)$ for all $t>0$.

When (2.3) is instead binding, $S_{b}^{*}(t)$ is a constant $S_{0}$ on the interval $\left[t_{1}, t_{2}\right]$ :

$$
\left.S_{b}^{*}(\theta, t)\right)=S_{b}^{*}\left(t_{1}\right)=S_{b}^{*}\left(t_{2}\right)=S_{0} \quad \forall t \in\left[t_{1}, t_{2}\right]
$$

where

$$
S_{b}^{*}\left(t_{i}\right)=S^{*}\left(t_{i}\right) \quad \forall i=1,2 .
$$

The optimality conditions requires that $\mu(t)$ is continuous at dates $t_{1}$ and $t_{2}$ which gives us:

$$
\mu\left(t_{1}\right)=\mu\left(t_{2}\right)=0 .
$$

Now, observe that (A.12) implies:

$$
-\dot{u}(t)-r C\left(S_{b}^{*}(\theta, t)\right) \exp (-r t)=\frac{d}{d t}(\mu(t) \exp (-r t)) .
$$


Integrating (A.15) over the interval $\left[t_{1}, t_{2}\right]$ gives us:

(A.16) $\int_{t_{1}}^{t_{2}} \frac{d}{d t}(\mu(t) \exp (-r t)) d t=0=u\left(t_{1}\right)-u\left(t_{2}\right)+r C\left(S_{0}\right)\left(\exp \left(-r t_{2}\right)-\exp \left(-r t_{1}\right)\right)$.

We deduce from (A.13) and (4.1) that necessarily (A.17) holds.

$$
t_{2}-t_{1}=\Delta
$$

Inserting into (A.16) and simplifying then yields (A.17).

Proof of Proposition 4. The proof borrows heavily from the proofs of Propositions 2 and 3 and is thus omitted. 